\title{
Two whitebacked planthopper resistance genes in rice share the same loci with those for brown planthopper resistance
}

\author{
GX Tan, QM Weng, X Ren, Z Huang, LL Zhu and GC He \\ Key Laboratory of the Ministry of Education for Plant Developmental Biology, College of Life Sciences, Wuhan University, Wuhan \\ 430072, People's Republic of China
}

\begin{abstract}
The whitebacked planthopper (WBPH), Sogatella furcifera, and brown planthopper (BPH) Nilaparvata lugens Stål are important sucking insects of rice (Oryza sativa L.) crops throughout the world. Rice 'B5', which has derived its resistance genes from the wild rice $O$. officinalis Wall ex Watt, is a line that is highly resistant to both WBPH and $\mathrm{BPH}$. Previously, two resistance genes against $\mathrm{BPH}, \mathrm{Qbp} 1$, and Qbp2 in 'B5' had been mapped onto chromosome 3 and chromosome 4 , respectively. In this study, we employed a mapping population composed of 187 recombinant inbred lines (RILs), produced from a cross between 'B5' and susceptible variety 'Minghui63', to locate the WBPH and $\mathrm{BPH}$ resistance genes. A RFLP survey of the bulked extremes from the RIL population identified two genomic regions, one on chromosome 3 and the other on chromosome 4 , likely containing the resistance genes to planthop-
\end{abstract}

pers. QTL analysis of the RILs further confirmed that two WBPH resistance genes were mapped on the same loci as Qbp1 and Qbp2, using a linkage map with 242 molecular markers distributed on 12 rice chromosomes. Of the two WBPH resistance genes, one designated $W b p h 7(t)$ was located within a 1.1-cM region between R1925 and G1318 on chromosome 3 , the other designated $W b p h 8(t)$ was within a $0.3-\mathrm{cM}$ region flanked by $\mathrm{R} 288$ and $\mathrm{S} 11182$ on chromosome 4. A two-way analysis of variance showed that two loci acted independently with each other in determining WBPH resistance. The results have significant implications in studying the interactions between sucking insects and plants and in breeding programs of resistance to rice planthoppers. Heredity (2004) 92, 212-217, advance online publication, 10 December 2003; doi:10.1038/sj.hdy.6800398

Keywords: sucking herbivore; planthopper resistance; gene mapping; recombinant inbred line; rice; Oryza officinalis

\section{Introduction}

Plants are challenged by a myriad of herbivorous insect attacks in their natural habitat. As a result, plants show various constitutive and induced defenses, protecting themselves from herbivore damage (Mauricio et al, 1997; Buell, 1998). Herbivorous insects also produce new foraging strategies to circumvent these defenses. The interactions between plants and herbivorous insects form a complex, co-evolved natural system.

Herbivores are generally classified as chewing insects and sucking insects. The most important sucking insects of rice (Oryza sativa L.) are planthoppers, including the whitebacked planthopper (WBPH), Sogatella furcifera, and the brown planthopper (BPH), Nilaparvata lugens Stål. These specialist herbivores use stylets to pierce plant cells, and consume large quantities of fluids as their nutritional source. Feeding by a large number of planthoppers causes drying of the leaves and wilting of the tillers, a phenomenon called 'hopperburn'. In addition, planthoppers cause indirect damage by acting as vectors of rice viruses. Several viral diseases such as rice grassy stunt viruses and rice ragged stunt viruses are transmitted by planthoppers (Anjaneyulu, 1986), and this causes additional damage.

Rice and planthoppers have become ideal models to study the interactions between plants and sucking herbivorous insects. Brown planthopper biotypes have been identified based on their abilities to feed and infest rice varieties with different resistance genes (IRRI, 1976). It was found that different species of planthoppers were overcome by the corresponding resistance genes of rice, which can even recognize different biotypes of the same insect species. Efforts have been made to collect resistance gene resources, and more than 10 resistance genes have been identified according to their reactions to different $\mathrm{BPH}$ biotypes and their locations on the chromosomes (Athwal et al, 1971; Lakshminarayana and Khush, 1977; Sidhu and Khush, 1979; Khush and Brar, 1991; Ishii et al, 1994; Huang et al, 2001; Yang et al, 2002).

For another specialist planthopper feeding on rice, the whitebacked planthopper, classical genetic analysis of selected rice accessions has identified six major $\mathrm{WBPH}$ resistance genes. They are Wbph1 (Sidhu and Khush, 1979), Wbph2 (Angeles et al, 1981), Wbph3 and wbph4 (Hernandez and Khush, 1985), Wbph5 (Wu and Khush, 1985), and Wbph6(t) (Li et al, 1990), respectively. Among these genes, wbph4 is a recessive resistance gene, whereas the other five are dominant. Some WBPH resistance 
genes have been incorporated into the improved varieties to broaden the genetic basis for resistance to WBPH, but none of them has been mapped onto the genetic map of rice.

Several wild species of Oryza are highly resistant to all the known biotypes of brown planthopper, whitebacked planthopper, and leafhopper (Jena and Khush, 1990). In transferring useful alien traits from wild species to cultivated rice, our laboratory has an interest in planthopper resistance genes, and has developed a series of novel breeding materials showing resistance to planthoppers, via wide hybridization between wild species and cultivated rice. We have assigned three resistance genes against $\mathrm{BPH}$ to these materials. One of the progeny lines, 'B5', which was introgressed from an accession of $O$. officinalis collected in China, conferred strong resistance to BPH (Shu et al, 1994; Yang et al, 1999). Two major resistance genes against BPH in 'B5' were assigned to the end of the long arm of chromosome 3 and to the short arm of chromosome 4 by genetic analysis of an $\mathrm{F}_{2}$ population (Huang et al, 2001). Recently, using a recombinant inbred line (RIL) population, we located another single dominant $\mathrm{BPH}$-resistant gene to the same region of chromosome 4 in 'B14', one of the progeny lines with strong resistance to BPH from O. latifolia (Yang et al, 2002). In addition to $\mathrm{BPH}$ resistance, we found that the introgression rice line ' $\mathrm{B} 5$ ' showed high resistance to WBPH. In the present study, we have mapped WBPH resistance genes using molecular-marker techniques in a RIL population. It was interesting to find that the resistance to WBPH was controlled by two genes, which were located to the same positions as the $\mathrm{BPH}$ resistance genes Qbp1 on chromosome 3 and Qbp2 on chromosome 4 , respectively. This paper presents the results in detail.

\section{Materials and methods}

\section{Plant materials and insects}

We used a mapping population composed of 187 RILs, which was developed through successive selfing for up to eight generations from a cross between 'B5', an introgression line of $O$. officinalis, and a cultivar 'Minghui63'. The WBPH used for infestation were collected from the rice fields in Hubei Province of China, and were maintained in the Genetics Institute of Wuhan University by feeding on plants of 'Taichung Native 1' (abbreviated as 'TN1'), a highly susceptible cultivar to WBPH. The BPH were obtained originally from the China Rice Research Institute (Huang et al, 2001).

\section{Evaluation of insect resistance}

Evaluation tests for $\mathrm{BPH}$ resistance were conducted in the summer of 2000, and those for WBPH resistance in 2001. The germinated seeds of RI lines were separately sown in a plastic tray $\left(52 \times 35 \times 10 \mathrm{~cm}^{3}\right)$ to assess the resistance. Each RI line was planted in one row across the width of the tray with 15 plants. The parents 'B5' and 'Minghui63', and the susceptible check 'TN1', were grown at random, together with RI lines in each tray. During the second leaf stage, the 2nd- to 3rd-instar nymphs of planthoppers were released to the trays for infestation at a density of 10 insects per seedling. When the seedlings of ' $\mathrm{TN1}$ ', the susceptible check row, were completely killed, we evaluated the severity scores of all the plants for each RI line, according to the criteria of Standard Evaluation System for Rice (IRRI, 1988). The resistance level of a RI line was then inferred based on the average score of the 15 seedlings. The tests were repeated three times for each insect species.

\section{Bulked segregant analysis}

According to the phenotypes of RILs, equal amounts of DNA from 18 highly resistant lines to WBPH were pooled to form the resistant bulk, and those from 20 susceptible lines were pooled as the susceptible bulk (Table 1). The DNA bulks were used together with the parental DNA for screening the molecular markers linked to the WBPH resistance in the bulked segregant analysis (Michelmore et al, 1991).

\section{Construction of the linkage map}

For construction of the genetic map of the RI population, we used molecular markers distributed across the 12 rice chromosome comprising 166 RFLPs, 52 SSRs, and 24 ESTs, which spanned approximately $1478 \mathrm{cM}$, with an average interval of $6.4 \mathrm{cM}$ between markers. Linkage of loci and mapped distances were determined by using Mapmaker/ Exp3.0 at an LOD score of 3.0 (Lincoln et al, 1992). Marker orders in the maps were in agreement with maps reported before (Causse et al, 1994; Harushima et al, 1998). QTL analysis of the $\mathrm{WBPH}$ and $\mathrm{BPH}$ resistance genes was conducted with QTLmapper 1.0 at a LOD threshold 2.4 as the criteria to indicate the QTL position (Wang et al, 1999).

\section{Results}

\section{Resistance score in RIL population}

The WBPH resistance phenotypes of the RILs were evaluated when the seedlings of the susceptible control 'TN1' were completely killed by WBPH at 12 days after infestation. The results indicated that the average severity scores of the parents 'B5' and 'Minghui63' were 2.1 and 8.5, respectively. 'B5' showed the trait of high resistance to WBPH. The severity scores of the 187 RILs infested with WBPH showed a continuous distribution, ranging from a low value of 1.0 to a high value of 8.5 , with an apparent peak around 5.0 in the distribution curve (Figure 1). Similarly, the BPH resistance score also

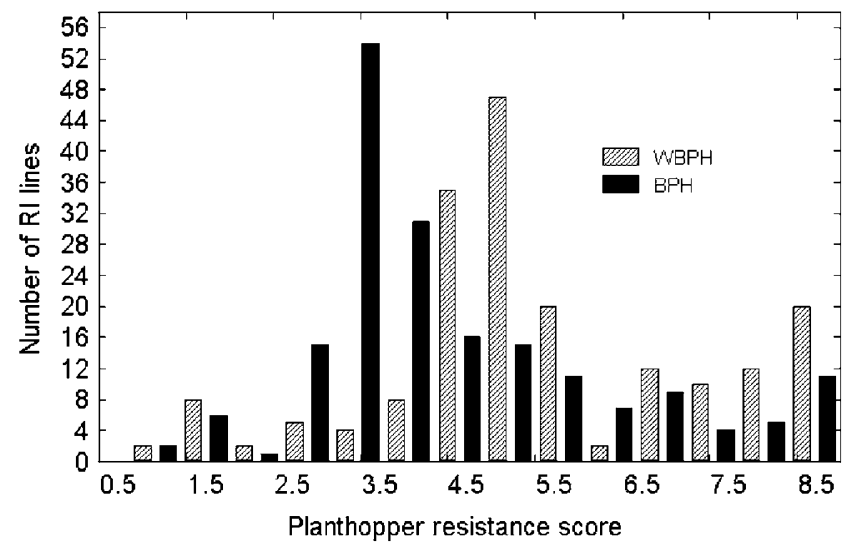

Figure 1 Distribution of respective $\mathrm{WBPH}$ and $\mathrm{BPH}$ resistance scores of the 187 RILs. The resistance scores are the averages of three independent tests 
showed a continuous distribution from 1.2 to 8.8 in the RIL population, with a more apparent peak at 3.5 . The high variance of this distribution shows that there are major genes controlling the segregation of $\mathrm{WBPH}$ and $\mathrm{BPH}$ resistance in the RIL population. The accessions selected from the RIL population to form the resistant and susceptible bulks were the same for the two insect species.

\section{Chromosomal positions of the resistance genes}

To detect linked markers and map WBPH resistance genes, 689 RFLP probes were employed for polymorphism screening in this study, and 178 (26\%) polymorphisms were detected between the parents ('B5' and 'Minghui63'). The survey of the two bulked extremes detected banding pattern differences with nine markers. Five of these were in a narrow region on chromosome 3 according to the linkage map that covers the whole rice genome. Another four markers were located in a contiguous region on chromosome 4 (Figure 2). The detection of these polymorphic markers indicated that each of the two genomic regions probably contained genes for WBPH resistance. As expected, the given RI

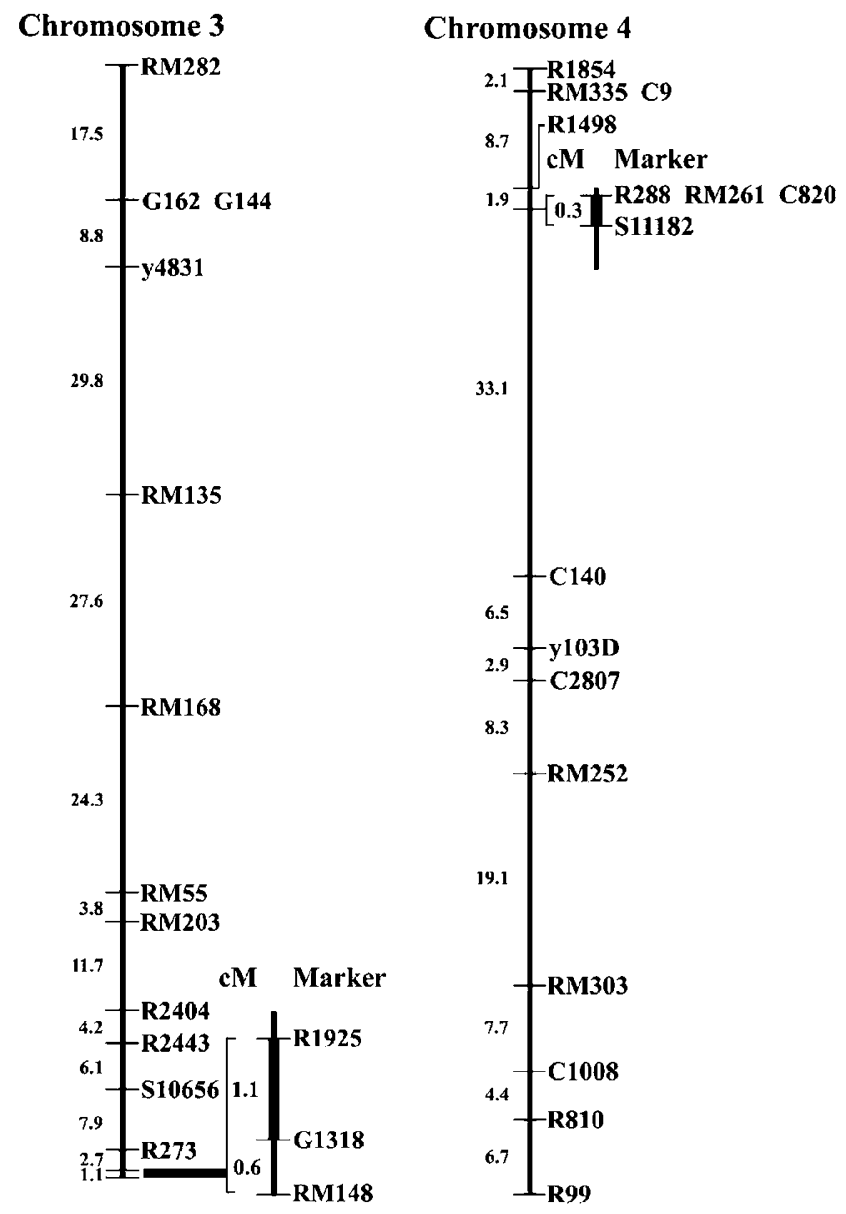

Figure 2 Location of two WBPH-resistant genes identified by QTL analysis. The right sides on chromosomes 3 and 4 show the magnification of the region related to WBPH-resistant genes, and the broadened bars indicate the loci where the WBPH-resistant genes were located. BPH-resistant genes were detected at the same genomic loci. line accessions of the two bulks of the $\mathrm{BPH}$ were in correspondence with those of the WBPH (Table 1); in the analysis of $\mathrm{BPH}$ resistance genes, cosegregation markers surrounding the narrow $Q b p 1$ and $Q b p 2$ regions were also detected, so that the two BPH-resistant genes on chromosome 3 and 4 were confirmed.

\section{Effects of WBPH and $\mathrm{BPH}$ resistance genes}

QTL analysis based on the data of the severity scores of RIL population confirmed the presence of the two WBPH resistance genes, and further determined their locations. One was mapped to a 1.1-cM length interval on the end of the long arm of chromosome 3, with an LOD score of 6.13, flanked by R1925 and G1318. This locus can explain $9.61 \%$ of the phenotypic variance of $\mathrm{WBPH}$ resistance in the population. We designated it as $W b p h 7(t)$. The other, designated as $W b p h 8(t)$, was resolved with an LOD score of 18.44 to a $0.3-c M$ interval between R288 and S11182 on the short arm close to the centromeric region of chromosome 4 (Figure 2). This locus accounted for $34.76 \%$ of the phenotypic variance of WBPH resistance in the population. The two QTLs jointly contributed $44.36 \%$ of the phenotypic variance of WBPH resistance in this population (Table 2). Also, the same resistance loci against BPH were detected on chromosome 3 and 4 by whole genome scanning, individually explaining 14.79 and $28.77 \%$ of the total variation in the RIL population, and collectively $43.56 \%$.

To assess the possible interactions between the two loci where the $\mathrm{WBPH}$ and $\mathrm{BPH}$ resistance genes resided, based on the genotypes of the marker locus lying closest to the peak in each of the two gene-containing genomic regions, a two-way analysis of variance was conducted. The analysis indicated that interactions between loci were not detected for WBPH resistance, but were significant for $\mathrm{BPH}$ resistance. However, this interaction effect was small relative to the main effects of the two $\mathrm{BPH}$ resistance loci marked by G1318 and S11182 (Table 3).

\section{Discussion}

A number of resistance genes against $\mathrm{WBPH}$ and $\mathrm{BPH}$ in rice have been reported in the literature. For example, cultivar 'N22' carries the resistance gene Wbph1 (Sidhu et al, 1979). Two genes, Wbph1 and Wbph2, govern resistance to WBPH in IR2035-117-3 (Angeles et al, 1981). 'IR26', the first BPH-resistant cultivar developed by the International Rice Research Institute, carries the $\mathrm{BPH}$ resistance gene Bph1. The cultivar 'IR36' carries bph2 (Athwal et al, 1971). All these indicate that most resistant cultivars carry one type of resistance gene, against either $\mathrm{WBPH}$ or $\mathrm{BPH}$. However, the resistant rice line ' $\mathrm{B} 5$ ', derived from wild rice with multiple resistances, confers high resistances to both $\mathrm{BPH}$ and $\mathrm{WBPH}$. To locate the resistance genes against two planthopper species, we have made efforts over the years to develop an RIL population from a cross between 'B5' and 'Minghui63', so that it becomes feasible to study the relationships between the genes controlling resistance to $\mathrm{WBPH}$ and those to $\mathrm{BPH}$ in one genetic system.

Herbivorous insect resistance is a trait that is very difficult to measure, as the scores vary with conditions of the plants and insects, and also the environments under which the tests are conducted. In order to guarantee the 
Table 1 Highly resistant and susceptible lines selected from RILs to form the two extreme bulks

\begin{tabular}{|c|c|c|c|c|c|}
\hline \multirow[t]{2}{*}{ Accession of resistant bulk } & \multicolumn{2}{|c|}{ Severity score $\pm S E$} & \multirow[t]{2}{*}{ Accession of susceptible bulk } & \multicolumn{2}{|c|}{ Severity score $\pm S E$} \\
\hline & WBPH & BPH & & WBPH & $B P H$ \\
\hline 24 & $3.4 \pm 1.9$ & $2.5 \pm 1.1$ & 15 & $8.5 \pm 0.8$ & $7.8 \pm 1.6$ \\
\hline 41 & $3.1 \pm 0.6$ & $2.0 \pm 1.4$ & 16 & $8.7 \pm 0.1$ & $8.6 \pm 0.3$ \\
\hline 67 & $1.7 \pm 1.5$ & $3.0 \pm 0.9$ & 17 & $8.8 \pm 0.4$ & $8.2 \pm 1.4$ \\
\hline 69 & $0.9 \pm 0.8$ & $3.0 \pm 0.9$ & 18 & $8.7 \pm 0.2$ & $8.5 \pm 0.1$ \\
\hline 77 & $1.2 \pm 0.6$ & $2.0 \pm 1.3$ & 21 & $8.8 \pm 0.1$ & $7.5 \pm 0.7$ \\
\hline 78 & $2.8 \pm 1.3$ & $3.0 \pm 1.1$ & 34 & $8.5 \pm 0.6$ & $8.3 \pm 0.7$ \\
\hline 90 & $2.6 \pm 1.1$ & $1.3 \pm 1.4$ & 37 & $8.8 \pm 0.3$ & $7.9 \pm 1.7$ \\
\hline 91 & $1.7 \pm 1.6$ & $3.1 \pm 0.5$ & 44 & $8.2 \pm 1.4$ & $8.8 \pm 0.3$ \\
\hline 93 & $1.6 \pm 1.3$ & $1.5 \pm 1.0$ & 64 & $8.8 \pm 0.3$ & $7.9 \pm 1.0$ \\
\hline 94 & $2.7 \pm 2.1$ & $3.0 \pm 0.6$ & 111 & $8.8 \pm 0.2$ & $8.5 \pm 0.5$ \\
\hline 110 & $2.5 \pm 2.0$ & $3.1 \pm 1.8$ & 113 & $8.9 \pm 0.1$ & $7.4 \pm 0.5$ \\
\hline 118 & $1.9 \pm 2.1$ & $2.0 \pm 0.9$ & 115 & $9.0 \pm 0.0$ & $8.3 \pm 0.9$ \\
\hline 126 & $2.7 \pm 0.3$ & $3.0 \pm 0.7$ & 119 & $9.0 \pm 0.0$ & $9.0 \pm 0.0$ \\
\hline 130 & $1.6 \pm 0.6$ & $3.1 \pm 0.9$ & 120 & $9.0 \pm 0.0$ & $9.0 \pm 0.0$ \\
\hline 131 & $1.5 \pm 0.9$ & $3.0 \pm 0.4$ & 129 & $8.4 \pm 1.0$ & $8.8 \pm 0.2$ \\
\hline 175 & $1.8 \pm 1.2$ & $2.1 \pm 1.1$ & 149 & $7.7 \pm 1.6$ & $9.0 \pm 0.0$ \\
\hline 176 & $2.5 \pm 1.8$ & $1.6 \pm 1.0$ & 155 & $8.7 \pm 0.4$ & $8.3 \pm 0.9$ \\
\hline \multirow[t]{3}{*}{177} & $1.7 \pm 0.8$ & $1.2 \pm 0.9$ & 159 & $8.3 \pm 1.2$ & $7.8 \pm 0.5$ \\
\hline & & & 162 & $8.1 \pm 1.1$ & $9.0 \pm 0.0$ \\
\hline & & & 169 & $8.7 \pm 0.2$ & $9.0 \pm 0.0$ \\
\hline
\end{tabular}

Table 2 Chromosomal locations of WBPH and BPH resistances

\begin{tabular}{|c|c|c|c|c|c|c|c|}
\hline \multirow[t]{2}{*}{ Flanking markers } & \multirow[t]{2}{*}{ Chrom. } & \multicolumn{2}{|c|}{$L O D$} & \multicolumn{2}{|c|}{ Var. explained \% } & \multicolumn{2}{|c|}{ Additive } \\
\hline & & WBPH & BPH & $W B P H$ & $B P H$ & WBPH & BPH \\
\hline R1925-G1318 & 3 & 6.13 & 9.75 & 9.61 & 14.79 & -0.504 & -0.624 \\
\hline R288-S11182 & 4 & 18.44 & 19.46 & 34.76 & 28.77 & -0.959 & -0.870 \\
\hline Total & & & & 44.36 & 43.56 & & \\
\hline
\end{tabular}

Table 3 A two-way analysis of variance of WBPH and BPH resistance in the RIL population based on genotypes of the marker locus from each of the two genomic regions

\begin{tabular}{|c|c|c|c|c|c|c|c|}
\hline \multirow[t]{2}{*}{ Effect } & \multirow[t]{2}{*}{$d f$} & \multicolumn{2}{|c|}{ MS } & \multicolumn{2}{|c|}{$F$} & \multicolumn{2}{|c|}{ P-level } \\
\hline & & WBPH & ВPH & $W B P H$ & BPH & WBPH & BPH \\
\hline 1(G1318) & 1 & 21.29 & 46.62 & 8.22 & 25.51 & 0.0047 & 0.0000 \\
\hline 2(S11182) & 1 & 148.42 & 98.52 & 57.30 & 53.92 & 0.0000 & 0.0000 \\
\hline $1 \times 2$ & 1 & 0.23 & 28.33 & 0.08 & 15.50 & 0.7667 & 0.0001 \\
\hline Error & 157 & 2.59 & 1.83 & & & & \\
\hline
\end{tabular}

accuracy of evaluation for resistance to planthoppers, it is necessary to carry out the evaluation experiments with repetitions. However, phenotypic evaluation of $F_{2}$ individuals does not allow for replicated trials. But RIL populations offer unique advantages for such a purpose. A well-characterized RIL population can be permanently propagated and used indefinitely without further genotyping, and phenotype evaluation can be replicated as needed. These advantages make a RIL population an ideal choice in studying insect resistance genes. The RIL population established between 'B5' and 'Minghui63' greatly facilitates the evaluation of resistances to different sucking insects and identification of resistance genes with high accuracy.

The evaluation experiments for $\mathrm{WBPH}$ resistance and $\mathrm{BPH}$ resistance were completed by different researchers.
The gene loci identified for $\mathrm{WBPH}$ and $\mathrm{BPH}$ resistances were detected based on the results of three independent evaluations of the RIL population. Our results further confirmed the $\mathrm{BPH}$ resistance gene locations in ' $\mathrm{B} 5$ ' that were determined previously using $\mathrm{F}_{2}$ populations (Huang et al, 2001; Wang et al, 2001). Although evaluations of resistances to WBPH and $\mathrm{BPH}$ and bulked segregant analysis were carried out by different researchers, the RI lines that were selected to form the extreme bulks happened to be the same. Thus, it was not surprising that the same set of the polymorphic molecular markers for resistance to $\mathrm{WBPH}$ and $\mathrm{BPH}$ were detected. Nevertheless, the polymorphic molecular markers in bulked segregant analysis did not assign resistance genes to concrete sites, but could only indicate the possible linkages of resistance genes with some 
specific chromosome regions. Interval mapping facilitates the assignment of resistance genes to precise positions. Our results of interval mapping showed that two WBPH resistance genes are located at the same loci with $\mathrm{BPH}$ resistance genes in a single mapping population composed of 187 RILs. Later, we accomplished a linkage map that covers the whole genome with RFLP, SSR, and EST markers; by a genome-wide scanning, two major resistant genes to both $\mathrm{WBPH}$ and $\mathrm{BPH}$ were identified on two different chromosomes. One site, containing Qbp1 and $W b p h 7(t)$, was mapped on the end of long arm of chromosome 3 and flanked by R1925 and G1318 within $1.1 \mathrm{cM}$. The other containing Qbp2 and $W b p h 8(t)$ was located between R288 and S11182 within $0.3 \mathrm{cM}$ on the short arm near the centromeric region of chromosome 4 .

It is an intriguing phenomenon in genetics as well as in breeding that several resistance genes against different species of planthoppers share the same loci. The extreme resistance and susceptibility of RILs to WBPH are consistent with those to $\mathrm{BPH}$, demonstrating that the two genes at each locus did not segregate in RIL population. Thereby, the results suggested that multiresistance genes exist in these two loci on chromosomes 3 and 4 , each of which can simultaneously confer both $\mathrm{WBPH}$ and $\mathrm{BPH}$ resistance. It has previously been shown that a single BPH-resistant gene can operate against different biotypes. The dominant gene Bph1 conferred resistance to biotypes 1 and 3 (Athwal et al, 1971). Two closely linked genes, Bph3 and bph4, conferred resistance to all the four biotypes (Sidhu and Khush, 1979). $B p h 10(t)$, derived from the wild species O. australiensis, showed resistance to biotypes 1, 2, and 3 (Ishii et al, 1994). Each of $Q b p 1, Q b p 2$, and Bph12(t) conferred resistance to biotypes 1 and 2 and to the field population in China (Huang et al, 2001; Yang et al, 2002). Similarly, it has been shown that one of the plant proteinase inhibitor (PI) genes can create resistance to several insect species. A good example was demonstrated by transferring a trypsin inhibitor gene from Vigna unguiculata to tobacco, where it conferred resistance to a wide range of herbivorous insects including lepidoptera, such as Heliothis and Spodoptera, coleoptera such as Diabrotica, Anthonomnous, and orthoptera such as Locusts (Hilder et al, 1987). Milligan et al (1998) isolated the Mi gene from tomato originally, due to its effect on pathogen root knot nematodes. But it was found later that the $M i$ gene also conferred resistance to the sucking insect aphid Macrosiphum euphorbiae. Thus, the Mi gene in tomato has dual specificity, showing resistance to both root knot nematodes and potato aphids (Rossi et al, 1998; Vos et al, 1998). All of these examples demonstrate that it is possible that plants achieve a broad range resistance simultaneously to different species of insects via a multi-resistance gene.

The interactions between herbivorous insects and plants are rather complex. There are some very interesting biological questions concerning how the resistances against WBPH and $\mathrm{BPH}$ are controlled by the same resistant genes, how rice-resistant varieties and susceptible varieties are recognized by different planthopper species and so forth. Identification of the molecular markers linked to genes simultaneously resistant to WBPH and BPH is helpful in cloning the resistance genes and ultimately elucidating the resistance mechanism against planthoppers. The results also provide an excellent opportunity to exploit ' $\mathrm{B} 5$ ' as a source of multiple resistances and to use the same set of markeraided selection in rice improvement to achieve resistance against different species of planthoppers.

\section{Acknowledgements}

We sincerely thank Dr T Sasaki and Dr SD Tanksley for kindly providing the RFLP probes. This study was supported by grants from the National Program of High Technology Development, and the National Special Program for Research and Industrialization of Transgenic Plants of China. The experiments reported in this paper were conducted in compliance with the current laws of the People's Republic of China.

\section{References}

Angeles ER, Khush GS, Heinrichs EA (1981). New genes for resistance to whitebacked planthopper in rice. Crop Sci 21: 47-50.

Anjaneyulu A (1986). Virus diseases of rice in India. Trop Agric Res Ser 19: 14-19.

Athwal DS, Pathak MD, Bacalangco E, Pura CD (1971). Genetics of resistance to brown planthoppers and green leaf hoppers in Oryza sativa L. Crop Sci 11: 747-750.

Buell CR (1998). Arabidopsis: a weed leading the field of plantpathogen interactions. Plant Physiol Biochem 36: 177-186.

Causse M, Fulton TM, Cho YG, Ahu SN, Chunwongse J, Wu K et al (1994). Saturated molecular map of the rice genome based on an interspecific backcross population. Genetics 138: 1251-1274.

Harushima Y, Yano M, Shomura A, Sato M, Shimano T, Kuboki $Y$ et al (1998). A high-density rice genetic linkage map with 2275 markers using a single $\mathrm{F}_{2}$ population. Genetics 148: 479-494.

Hernandez JE, Khush GS (1985). Genetics of resistance to whitebacked planthopper in some rice (Oryza sativa L.) varieties. Oryza 18: 44-50.

Hilder VA, Gatehouse AMR, Sheerman SE, Barker RF, Boulter D (1987). A novel mechanism of insect resistance engineered into tobacco. Nature 300: 160-163.

Huang Z, He GC, Shu LH, Li XH, Zhang QF (2001). Identification and mapping of two brown planthopper resistance genes in rice. Theor Appl Genet 102: 929-934.

IRRI (1976). IRRI Annual Report for 1975, International Rice Research Institute: Los Banõs, Philippines.

IRRI (1988). Standard Evaluation System for Rice, International Rice Research Institute: Manila, Philippines.

Ishii T, Brar DS, Multani DS, Khush GS (1994). Molecular tagging of genes for brown planthopper resistance and earliness introgressed from Oryza australiensis into cultivated rice, O. sativa. Genome 37: 217-221.

Jena KK, Khush GS (1990). Introgression of genes from Oryza officinalis Well ex Watt to cultivated rice, O. sativa L. Theor Appl Genet 80: 737-745.

Khush GS, Brar DS (1991). Genetics of resistance to insects in crop plants. Adv Agron 45: 223-274.

Lakshminarayana A, Khush GS (1977). New genes for resistance to the brown planthopper in rice. Crop Sci 17: 96-100.

Lincoln S, Daly M, Lander ES (1992). Constructing genetic maps with MAPMAKER/EXP 3.0, Whitehead Institute Technical Report, 2nd edn. Whitehead Institute Cambridge: Massachusetts, USA.

Li XM, Xiong ZM, Min SK, Hu GW (1990). Genetical analysis of resistance to whitebacked planthopper Sogatella furcifera (Horvath) in four rice varieties (Oryza sativa L.) of Yunan province. Chin J Rice Sci 4: 113-116. 
Mauricio R, Rausher MD, Burdick DS (1997). Variation in the defense strategies of plants: are resistance and tolerance mutually exclusive? Ecology 78: 1301-1311.

Michelmore RW, Paran I, Kesseli RV (1991). Identification of markers linked to disease resistance genes by bulked segregant analysis: a rapid method to detect markers in specific genomic regions by using segregating populations. Proc Natl Acad Sci USA 88: 9828-9832.

Milligan SB, Bodeau J, Yaghoobi J, Kaloshian I, Zabel P, Williamson VM (1998). The root knot nematode resistance gene $M i$ from tomato is a member of the leucine zipper, leucleotide binding, leucine-rich repeat family of plant genes. Plant Cell 10: 1307-1320.

Rossi M, Goggin FL, Milligan SB, Kaloshian I, Ullman DE, Williamson VM (1998). The nematode resistance gene $M i$ of tomato confers resistance against the potato aphid. Proc Natl Acad Sci USA 95: 9750-9754.

Shu LH, Liao LJ, Wan HQ (1994). Genetic analysis and breeding effect for resistance to bacteria in wild rice. J Wuhan Univ (Nat Sci Ed) 3: 95-100.

Sidhu GS, Khush GS (1979). Linkage relationships of some genes for disease and insect resistance and semidwarf stature in rice. Euphytica 28: 233-237.
Sidhu GS, Khush GS, Medrano FG (1979). A dominant gene in rice for resistance to whitebacked planthopper and its relationship to other plant characteristics. Euphytica 28: 227-232.

Vos P, Simons G, Jesse T, Wijbrandi J, Heinen L, Hogers R et al (1998). The tomato Mi-1 gene confers resistance to both rootknot nematodes and potato aphids. Nat Biotechnol 16: 1365-1369.

Wang BN, Huang Z, Shu LH, Ren X, Li XH, He GC (2001). Mapping of two new brown planthopper resistance genes from wild rice. Chin Sci Bull 46: 1092-1095.

Wang DL, Zhu J, Li ZK, Paterson AH (1999). A Computer Software for Mapping Quantitative Trait Loci (QTLs) with Additive Effects, Epistatic Effects and QTL $\times$ Environment Interactions, QTLMapper Version 1.0. Zhe Jiang University, China.

Wu CF, Khush GS (1985). A new dominant gene for resistance to whitebacked planthopper in rice. Crop Sci 25: 505-509.

Yang CJ, Yang ZH, Hu JF, He GC, Shu LH (1999). Study on the brown planthopper resistance in introgressive lines from wild rice. Acta Phytophylacica Sin 26: 197-202.

Yang HY, Ren X, Weng QM, Zhu LL, He GC (2002). Molecular mapping and genetic analysis of a rice brown planthopper (Nilaparvata lugens Stål) resistance gene. Hereditas 136: 39-43. 